\title{
Prototipo de un oxímetro de pulso con ESP8266 Wi-Fi
}

\author{
Gabriel Contreras Mota ${ }^{1}$, Rafael Lemuz López¹, Carlos Guillén Galván², \\ Blanca Bermúdez Juárez ${ }^{1}$ \\ ${ }^{1}$ BUAP, Facultad de Ciencias de la Computación, \\ Puebla, México \\ ${ }^{2}$ BUAP, Facultad de Ciencias Físico Matemáticas, \\ Puebla, México
}

gabriel.contreras.217@gmail.com,rafalemuz@cs.buap.mx, cguillen@fcfm.buap.mx, bbj.solarium.cs.buap.mx

\begin{abstract}
Resumen. En este trabajo se describe el diseño y construcción de un oxímetro de pulso con conexión WIFI. El dispositivo se conecta a un servidor que muestra la saturación de oxígeno y la frecuencia cardíaca. La información se visualiza en una aplicación web. El dispositivo se basa en el microcontrolador ESP8266 que establece la conexión inalámbrica y el envío de la información al servidor. En el artículo se describe el diseño del dispositivo y se muestran algunos resultados experimentales.
\end{abstract}

Palabras clave: Oxímetro de pulso, WiFi, microcontrolador ESP8266, aplicación web.

\section{Prototype of a Pulse Oximeter with ESP8266 Wi-FI}

\begin{abstract}
This paper describes the design and construction of a pulse oximeter with WIFI connection. This device connects to a server that shows oxygen saturation and heart rate. The information is displayed on a web application. The device is based on the ESP8266 microcontroller which establishes the wireless connection and sends the information to the server. In this article, the design of this device is described and some experimental results are shown.
\end{abstract}

Keywords: Pulse oximeter, WiFi, ESP8266 microcontroller, web application.

\section{Introducción}

El oxímetro de pulso es un dispositivo médico utilizado en el monitoreo no invasivo de la frecuencia cardiaca y el porcentaje de saturación de oxigeno ( $\mathrm{SpO} 2)$ en la sangre. La Oximetría de Pulso ha evolucionado en buena medida porque los dispositivos frecuentemente reducen su tamaño considerablemente permitiendo el desarrollo de 
equipos portátiles e incrementando la distribución y su uso en el monitoreo del oxígeno en la sangre.

El oxígeno es uno de los elementos indispensables para la vida, el porcentaje normal en el cuerpo varia de $95 \%$ a $100 \%$ [1], teniendo algunas variaciones dependiendo de la altura sobre el nivel del mar en la que se encuentre, bajos niveles de oxigenación en la sangre generan una condición llamada Hipoxemia. La Hipoxemia puede ser provocada por diferentes causas, entre las más frecuentes se encuentran las enfermedades pulmonares o problemas cardiacos, una atención rápida es crucial para la salud del paciente, el uso del Oxímetro de Pulso proporciona una medición en tiempo real del estado del paciente facilitando su tratamiento.

La Oximetría es ampliamente utilizada en las intervenciones quirúrgicas, específicamente en el control de la anestesia, en el diagnóstico y tratamiento de enfermedades de obstrucción pulmonar crónica (EPOC), el control de la gravedad asma, así como Infecciones respiratorias como la influenza y la neumonía. En México desde el 2009 después del brote epidemiológico de influenza mundial, los casos de influenza han estado presente año con año, en el periodo de 2015-2016 se confirmaron 870 casos [2], el uso de la Oximetría de Pulso proporciona un método en el diagnóstico de los casos que se requiera la hospitalización del paciente debido a su bajo nivel de saturación de oxígeno en la sangre.

El internet de las cosas (IoT) ha intensificado su desarrollo en el último año, dispositivos cotidianos están ahora conectados a internet pudiendo ser controlados de manera remota. Esta misma tecnología puede ser aplicada a la medicina, el monitoreo en tiempo real del paciente y su entorno, así como su seguimiento posterior al alta hospitalaria traerían un beneficio en la salud del paciente.

Consultado el estado del arte sobre el tema encontramos el trabajo presentado por el Centro de Bioingeniería de Colombia, demuestra que la sustitución de elementos analógicos disminuye en gran medida el consumo eléctrico, el cambio de filtros analógicos a digitales y el uso de PWM en el control de los diodos emisores, su sistema se basa en tarjeta y sondas prediseñadas para uso médico [3].

La propuesta presentada por el grupo de investigación de la Universidad de Colombia implementa un sistema de pulsioximetría y capnografía en un microcontrolador PSoC, el cual utiliza filtros digitales para el corte de frecuencia, así mismo mantiene una conexión inalámbrica tipo TCP/IP a un dispositivo Android el cual se encarga del almacenamiento y visualización [4]. La investigación realizada por Instituto Politécnico Nacional de México destaca la utilidad de implementar la conexión inalámbrica, así mismo el uso de la comunicación TCP/IP. La implementación del estándar HL7 en la comunicación del dispositivo con el servidor garantiza la compatibilidad de los datos con otros dispositivos de medición, es sistemas está basado en una PIC con un módulo WiFi, la trasmisión de datos se centra en el sistema hospitalario [5].

El objetivo de este trabajo es el desarrollo de un prototipo de un Oxímetro de Pulso y aplicación web que solucione el problema del monitoreo de pacientes a distancia, se busca que el dispositivo obtenga la señal cardiaca y de la saturación de oxigeno de la sangre del paciente, el instrumento por sí mismo será capaz de establecer una conexión a un servidor remoto al cual enviara la señal obtenida, posteriormente la aplicación 
calculara el nivel de oxígeno y el pulso cardiaco mostrándolas en pantalla al igual que la curva pletismográfica; se emplean las tecnologías existentes, utilizando la menor cantidad de componentes.

\section{Componentes y construcción}

La oximetría de pulso se basa en la estimación de la saturación de oxígeno en la sangre por medio de la espectrofotometría que es la medición de la cantidad de energía radiante que puede absorber un elemento, en este caso la hemoglobina presente en la sangre. La estructura principal del dispositivo se basa en el esquema que se muestra en la Fig. 1, se maneja cuatro etapas para resolver el problema.

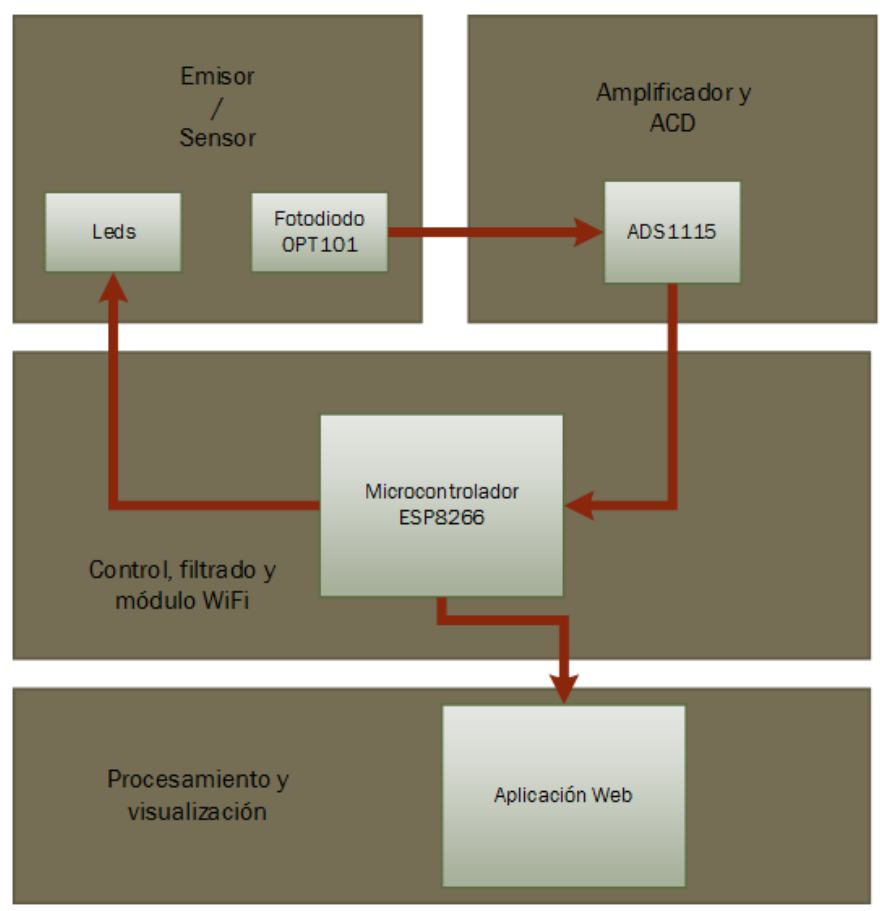

Fig. 1. Esquema de la absorción de la luz.

\subsection{Emisor de luz y sensor}

La hemoglobina es una proteína que se genera en la médula ósea, está presente en los glóbulos rojos y es la encargada del trasporte de $\mathrm{O} 2$ a cada rincón del cuerpo y regresando el $\mathrm{CO} 2$ de la sangre a los pulmones para su expulsión. La hemoglobina tiene diferentes niveles de absorción de la luz al transportar $\mathrm{O} 2$ y $\mathrm{CO} 2$ permitiendo su medición a través de la diferencia que hay entre estos dos a diferentes longitudes de onda. 
Oxihemoglobina se denomina a la hemoglobina que trasporta O2, esta absorbe mayor cantidad de luz roja (640-660nm) y deja pasar la luz infrarroja (910-940nm). Por su parte, la desoxihemoglobina trasporta $\mathrm{CO} 2$ y absorbe la luz infrarroja, pero deja pasar la luz roja. En la Fig.2, se muestra la absorción de la oxihemoglobina y desoxihemoglobina a diferentes longitudes de onda, como se puede observar el intervalo de $640 \mathrm{~nm}$ a $940 \mathrm{~nm}$ es el segmento que presenta la mayor diferencia de absorción de luz.

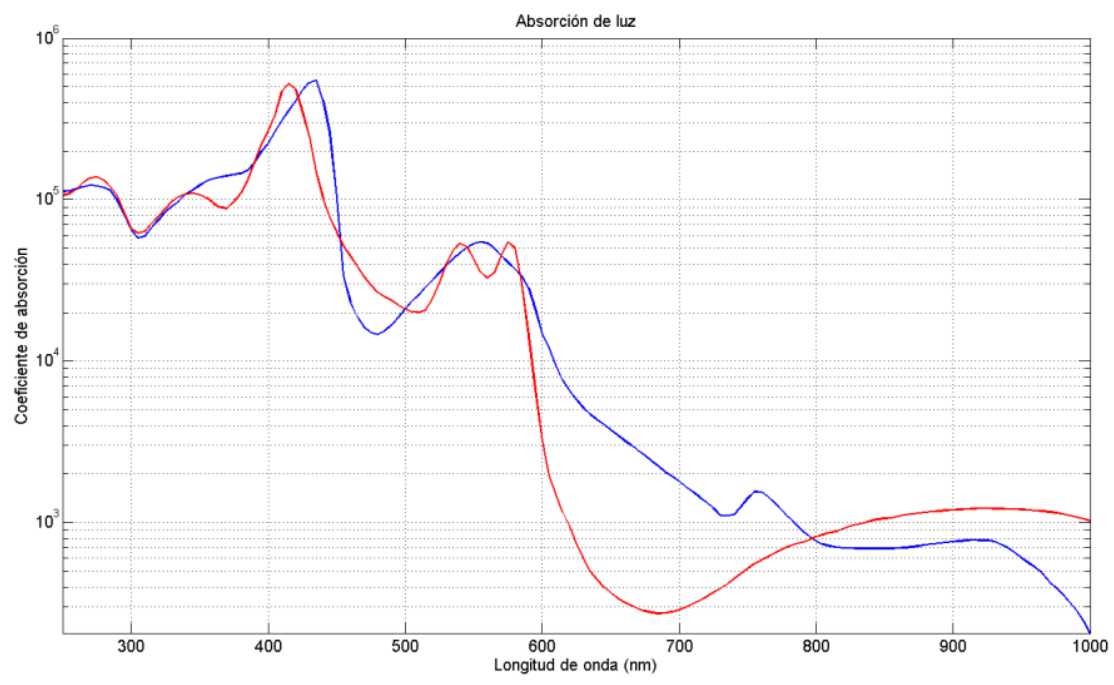

Fig. 2. Esquema de la absorción de la luz.

El funcionamiento del oxímetro de pulso se basa en lo anteriormente mencionado, utilizando dos emisores de luz, en este caso diodos lumínicos con longitudes de onda $660 \mathrm{~nm}$ (rojo) y 940nm (infrarrojo), se sincronizan secuencialmente por medio del microcontrolador con un fotodetector.

El emisor consta de un led rojo con longitud de onda de $660 \mathrm{~nm}$ y un led infrarrojo con longitud de onda de 940nm aproximadamente, ambos leds serán controlados por un pulso sincronizado con el fotodiodo, en la Fig. 3 vemos es esquema dela colocación de los diodos emisores y el fototransistor, en el cual las ondas de luz de cada longitud atraviesan el dedo y estas tendrán una variación de acuerdo a la hemoglobina.

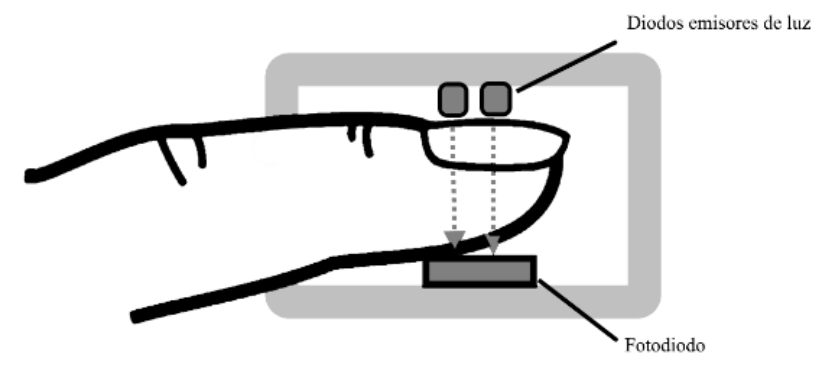

Fig. 3. Funcionamiento del sensor. 
Utilizamos el componente OPT101, es un fotodiodo monolítico, tiene un rango de espectro promedio de 400 a $1100 \mathrm{~nm}$, además cuenta con un amplificador de transimpedancia, la Fig. 4 vemos el diagrama del fotodiodo, se utiliza la configuración típica proporcionada por el fabricante administrando un voltaje de 3.3 volts [6].

\subsection{Amplificador y ACD}

La salida del fotodiodo es amplificada y convertida de analógico a digital por el componente ADS1115. Este convertidor digital(ADC) tiene una resolución de 16bits de los cuales solo 15bit serán utilizados y que un bit es utilizado por el signo, se manejaran 32768 valores para la señal. Cuanta con un PGA que amplifica la ganancia del sensor para su conversión, este amplificador tiene 6 niveles de ganancia programable desde el dispositivo, la comunicación de este componente al microcontrolador se lleva a cabo por el bus de datos I2C, en la Fig. 4, se muestran los esquemas del componente [7].
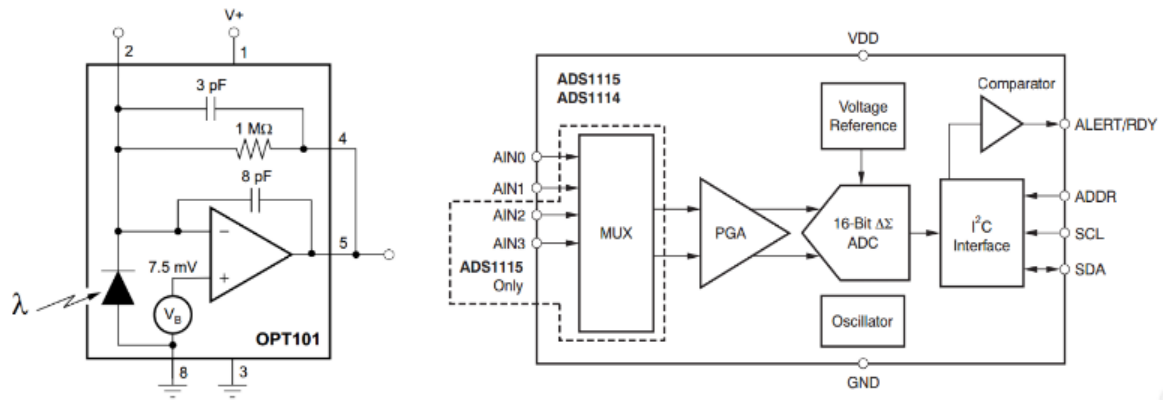

Fig. 4. Esquema del fotodiodo y del convertidor digital.

\subsection{Control, filtrado y trasmisor de la señal procesada}

El microcontrolador en este caso el ESP8266 será el que se encargue de realizar las tres tareas, controlar los diodos emisores, filtrar la señal recibida por el convertidor digital y finalmente trasmitir la señal resultante al servidor de la aplicación web. El microcontrolador ESP8266 es un chip de bajo costo de la marca Espressif, es un módulo Wi-Fi que puede realizar conexiones TCP/IP, en la Fig. 5 se observa el dispositivo, así mismo el esquemático de las entradas y salidas del dispositivo; principalmente el dispositivo fue diseñado para trabajar junto a un microcontrolador [8], posteriormente la empresa libero un SDK que le permitía al dispositivo ser programado; entre sus características principales son:

- CPU Tesilica Xtensa LX106 de 32-bit de 160MHz

- $\quad$ 96k de DRAM,64k de IRAM

- $4 \mathrm{mb}$ de memoria Flash

- $\quad$ Stack TCP/IP

- 100 metros de alcance

- Protocolos $802.11 \mathrm{~b} / \mathrm{g} / \mathrm{n}$ 


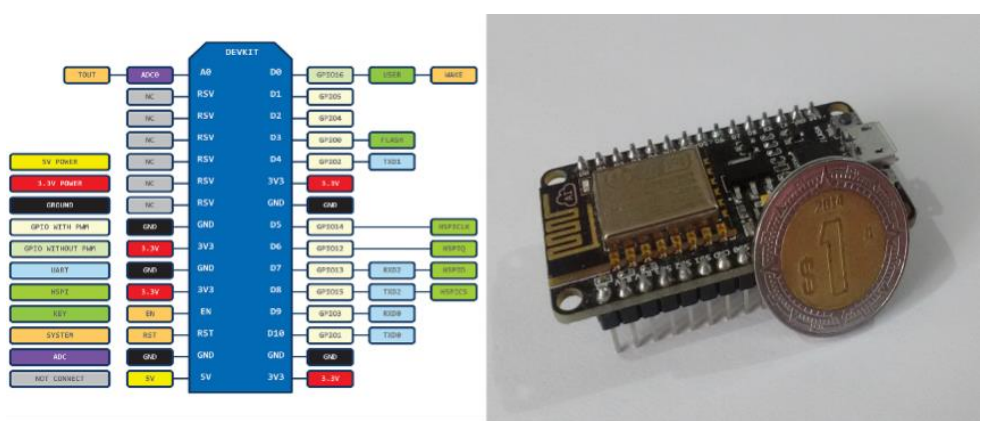

Fig. 5. Diagrama de conexión ESP8266-12.

El elemento a destacar de este microcontrolador es la arquitectura a 32bits, que permite la manipulación de datos de gran tamaño. El dispositivo puede ser programado de dos formas: interpretado, en este caso se manejan los lenguajes LUA y MicroPython y Compilado a partir de la API de Arduino que se basa en un lenguaje simplificado de $\mathrm{C} / \mathrm{C}++$ con su compilador y procesamiento basado en Java. Para mayor velocidad se utiliza el compilador nativo Gcc.

Para obtener la medición de la hemoglobina el microcontrolador controla por medio de pulsos en encendido y apagado de los diodos emisores, estos tendrán un tiempo de encendido de 50us seguidos de un desfase de 450us en el cual el sensor podrá obtener la medición.

Una vez obtenidos los datos de las mediciones de las dos ondas de luz, se filtra las señales por medio de un filtro digital, utilizamos un filtro pasa-banda IIR, que tiene una carga computacional menor, la frecuencia de corte se establece entre $0.5 \mathrm{~Hz}$ a $5 \mathrm{~Hz}$. Su función es limitar la frecuencia al rango de las mediciones, en este caso las pulsaciones del corazón, así, el rango va de 30 a 300ppm, señales mayores a 5 Hz significarán ruido en la señal, principalmente generado por el movimiento del paciente, en la Fig. 6 podemos observar las dos diferentes señales en RAW y su posterior filtrado. Este filtro se rige por la formula siguiente:

$$
H(z)=\frac{\sum_{k=0}^{N} b_{k} z^{-k}}{1+\sum_{k=1}^{M} a_{k} z^{-k}}
$$

Obtenida la señal digital es filtrada eliminando ruido y picos por el movimiento del paciente, se procede al envío de esta señal al servidor, el dispositivo se conecta a una red inalámbrica con conexión a internet, una vez establecida la conexión a internet se conecta al servidor listo para proceder al envió.

\subsection{Procesamiento y visualización}

Para que el monitoreo del paciente pueda ser seguido en cualquier parte sin la necesidad que este se traslade, la aplicación web será el medio por el cual se procesara y visualizara esta información. La base principal será el servidor el cual se creó con Node js, el oxímetro desarrollado a partir del microcontrolador ESP8266 servirá a la ves como cliente con el servidor en el intercambio de los datos a través del protocolo 
HTTP. Teniendo los datos de la medición del paciente la aplicación procesara para obtener la saturación de oxígeno y la frecuencia cardiaca, además dejara la señal intacta para su visualización. Debido a la variación de la oxihemoglobina y desoxihemoglobina con respecto a la absorción de la luz, se puede calcular el porcentaje de saturación de oxígeno conociendo la cantidad relativa de cada longitud de onda a través siguiente fórmula:

$$
\% \text { SaturaciondeOxigeno }=\frac{\text { Oxihemoglobina }}{\text { Oxihemoglobina }+ \text { Desoxiheoglobina }} * 100
$$

$\mathrm{Al}$ tomar estas mediciones ambas señales pasan a través de la piel, tejido, hueso, etc. A esto se le llama componente estática (DC) ya que no pulsan en comparación a la sangre arterial conocida como la parte dinámica (AC), en la Fig. 6, se observa el esquema de las diferentes capas del dedo.

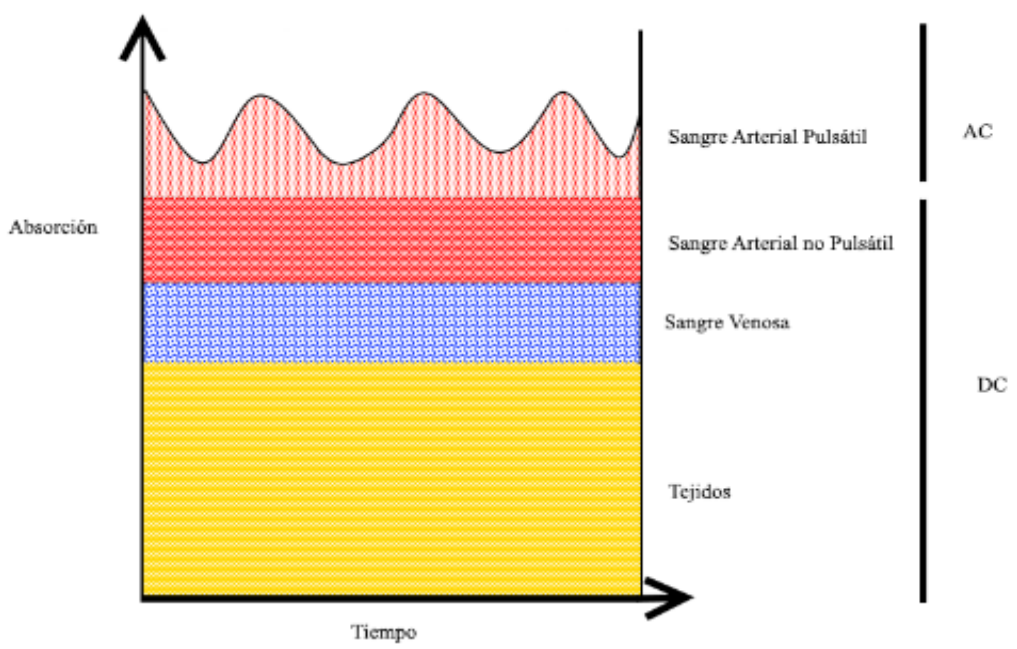

Fig. 6. Absorción de la luz de los componentes del dedo.

Debido a esto para obtener una medición correcta la saturación de oxigeno se calcula el cociente entre el valor de cada muestra con la siguiente formula:

$$
R=\frac{\frac{\text { ACrojo }}{\text { DCrojo }}}{\frac{\text { ACinfrarrojo }}{\text { DCinfrerrojo }}}
$$

Teniendo las dos señales en los rangos previamente establecidos, aplicamos la ecuación 2, obtenemos la razón, se establece el porcentaje a través de la curva generada mediante un proceso de calibración. En cuanto al pulso cardiaco solo restaría aplicar la trasformada rápida de Fourier en base a la frecuencia de salida.

El valor de R se encuentra ligado al porcentaje de $\mathrm{SpO} 2$, una vez calibrado el equipo, estos datos de comparación son guardados en el microcontrolador para su uso. El monitoreo en tiempo real se lleva a cabo accediendo a la página, una vez abierta esta 
generará peticiones al servidor, en pantalla se muestra los niveles de oxígeno en la sangre, así como la frecuencia cardiaca, en la parte posterior se presenta la onda pletismográfica de la medición.

\section{Resultados}

El dispositivo se montó en una tarjeta de pruebas, la alimentación del dispositivo se realiza por medio de 5volts, el microcontrolador en la tarjeta que está montada lleva consigo un regulador de tensión a 3.3volt el cual alimenta a los demás dispositivos. En la Fig. 8 se puede ver los componentes ya funcionando.

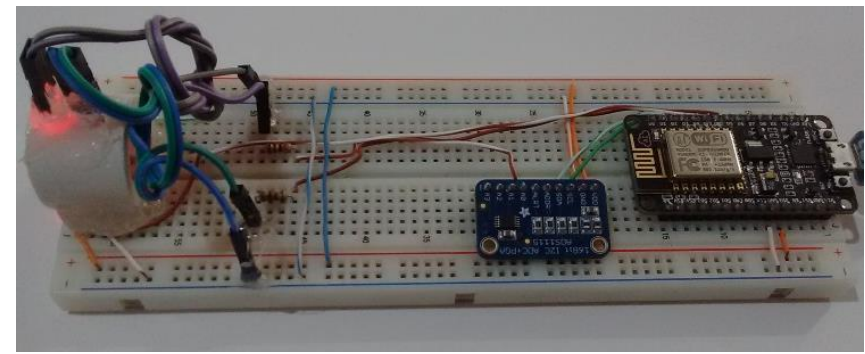

Fig. 7. Prototipo oxímetro de pulso.

Al obtener la señal del paciente se procedió a verificar el resultado final del filtrado, lo que se busca es eliminar ruido generado por el mismo dispositivo, por la luz ambiental y por los movimientos que el paciente llegara a producir, las mediciones fueron enviadas por medio de puerto serial a un programa de Matlab el cual grafica las señales, se compararon se las señales con y sin filtro como se observa en la Fig. 8.

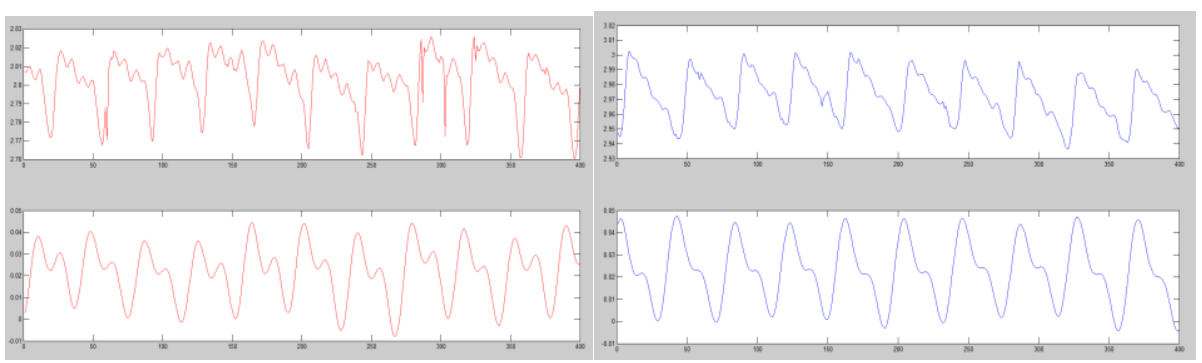

Fig. 8. Señal emisor IR (color azul) y señal emisor rojo (color rojo), la gráfica superior corresponde a la captura sin procesar y la inferior después de aplicar el filtrado.

La conexión del dispositivo a la red inalámbrica local y al servidor de la aplicación web se mantuvo estable en las pruebas realizadas con mediciones reales a periodos de 6 horas continuas por 7 días, los datos recibidos por el servidor fueron almacenados en archivos JSON, al identificar la dirección MAC del dispositivo se asigna al paciente, los archivos guardan la fecha y hora de la medición. 


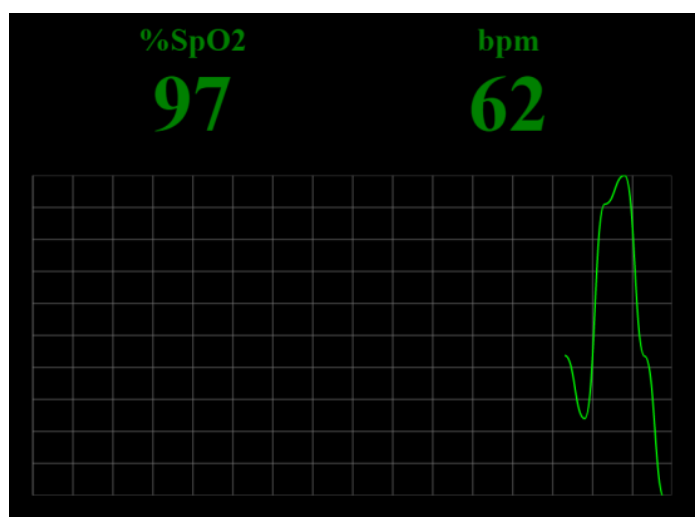

Fig. 9. Monitoreo en tiempo real.

En la Fig. 9 se muestra la captura de la visualización de la aplicación web, esta misma está configurada a modo responsivo por lo que el usuario podrá visualizarla en cualquier dispositivo.

\section{Conclusión}

En este documento se presentó el prototipo de un Oxímetro de Pulso basado en el microcontrolador ESP8266con conexión WiFi, el cual permite monitorear a distancia de la saturación de oxígeno y el pulso cardiaco, se logró mantener un monitoreo continuo, además de guardar un registro del mismo para su posterior análisis. En un futuro se busca la implementación de este sistema a otros signos vitales.

Agradecimientos. Los autores agradecemos el apoyo financiero del proyecto de red PRODEP, modelación matemática y computacional en medicina y dinámica poblacional.

\section{Referencias}

1. Mejía, H., Mejía, M.: Oximetría de pulso. Revista de la Sociedad Boliviana de Pediatría, Vol. 51, No. 2 (2012)

2. Cuitláhuac, M.: Actualización de la situación de influenza durante la temporada 20152016. Boletín Epidemiológico, Sistema Nacional de Vigilancia Epidemiológica, México, Vol. 33, No. 6, pp. 4-9 (2016)

3. Pérez, R. P., Pérez, J., Betancur, M. J., Bustamante, J.: Método alternativo del control de la intensidad lumínica para pulsioximetría en tiempo real. Ing. Biomed., Vol. 9, No. 17 (2015)

4. Gómez, C. A., Velasco, J.: Sistema de pulsioximetría y capnografía para dispositivos móviles Android. Ing. Biomed., Vol. 8, No. 15 (2014)

5. Ochoa, V., Sandoval, R.: Design of pulse oximeter with Wifi connectivity and interoperability with standard HL7 and IEE 1107.10404:2008. Systemics, Cybernetic And Informatics, Vol. 12, No. 7 (2014) 
Gabriel Contreras Mota, Rafael Lemuz López, Carlos Guillén Galván, Blanca Bermúdez Juárez

6. Texas Instruments Incorporated: OPT101 Monolithic Photodiode and Single-Supply Trans-impedance Amplifier (2015)

7. Texas Instruments Incorporated: Ultra-Small, Low-Power, 16-Bit Analog-to Digital Converter with Internal Reference ADS1115 (2009)

8. Espressif System: ESP8266EX Datasheet (2016) 